\title{
Operant Conditioning of a Spinal Reflex Can Improve Locomotion after Spinal Cord Injury in Humans
}

\author{
Aiko K. Thompson, ${ }^{1,2,3,5}$ Ferne R. Pomerantz, ${ }^{1,4}$ and Jonathan R. Wolpaw ${ }^{1,2,3,5}$ \\ ${ }^{1}$ Helen Hayes Hospital, New York State Department of Health, West Haverstraw, New York 10993, ${ }^{2}$ Wadsworth Center, New York State Department of \\ Health, Albany, New York 12201-0509, ${ }^{3}$ Department of Neurology, Neurological Institute, and ${ }^{4}$ Department of Rehabilitation and Regenerative Medicine, \\ Columbia University, New York, New York 10032, and 5Department of Biomedical Sciences, State University of New York, Albany, New York 12222
}

Operant conditioning protocols can modify the activity of specific spinal cord pathways and can thereby affect behaviors that use these pathways. To explore the therapeutic application of these protocols, we studied the impact of down-conditioning the soleus $\mathrm{H}$-reflex in people with impaired locomotion caused by chronic incomplete spinal cord injury. After a baseline period in which soleus H-reflex size was measured and locomotion was assessed, subjects completed either $30 \mathrm{H}$-reflex down-conditioning sessions (DC subjects) or 30 sessions in which the H-reflex was simply measured [unconditioned (UC) subjects], and locomotion was reassessed. Over the 30 sessions, the soleus H-reflex decreased in two-thirds of the DC subjects (a success rate similar to that in normal subjects) and remained smaller several months later. In these subjects, locomotion became faster and more symmetrical, and the modulation of EMG activity across the step cycle increased bilaterally. Furthermore, beginning about halfway through the conditioning sessions, all of these subjects commented spontaneously that they were walking faster and farther in their daily lives, and several noted less clonus, easier stepping, and/or other improvements. The H-reflex did not decrease in the other DC subjects or in any of the UC subjects; and their locomotion did not improve. These results suggest that reflex-conditioning protocols can enhance recovery of function after incomplete spinal cord injuries and possibly in other disorders as well. Because they are able to target specific spinal pathways, these protocols could be designed to address each individual's particular deficits, and might thereby complement other rehabilitation methods.

\section{Introduction}

The primary goal of neurological rehabilitation is to restore important motor and cognitive skills that have been impaired by injury or disease. Current therapeutic methods consist primarily of the repeated practice of these skills (e.g., treadmill locomotion, reach and grasp actions) (Wernig and Müller, 1992; Edgerton et al., 1997, 2001, 2008; Harkema et al., 1997; Taub et al., 1999; Wernig et al., 2000; Maegele et al., 2002; Taub and Uswatte, 2003; Wolf et al., 2006), with the expectation that this practice will lead to plasticity that improves function (Koski et al., 2004; Thickbroom et al., 2004; Thomas and Gorassini, 2005; Yen et al., 2008). Although this strategy is logical and often beneficial, it is seldom completely successful. Novel strategies that can complement current methods and thereby enhance skill restoration are needed.

\footnotetext{
Received Aug. 20, 2012; revised Dec. 6, 2012; accepted Dec. 10, 2012

Author contributions: A.K.T. and J.R.W. designed research; A.K.T. performed research; A.K.T. analyzed data; A.K.T., F.R.P., and J.R.W. wrote the paper.

This work was supported by the New York State Spinal Cord Injury Research Trust (Grant C023685 to A.K.T.); the National Institutes of Health (Grants NS69551 to A.K.T., NS22189 to J.R.W., and NS061823 to J.R.W. and Xiang Yang (hen); Helen Hayes Hospital Volunteer Corp (to A.K.T.); and the Helen Hayes Hospital Foundation (to A.K.T.). We thank Drs. Jonathan S. Carp, Dennis J. McFarland, Richard L. Segal, Richard B. Stein, and Elizabeth Winter Wolpaw for helpful comments on the manuscript; Elaine DeFrancesco for clinical assistance; and Briana M. Abel for assistance in data collection.

The authors declare no competing financial interests.

Correspondence should be addressed to Dr. Aiko K. Thompson, Helen Hayes Hospital, Route 9W, West Haverstraw, NY 10993. E-mail: thompsona@helenhayeshosp.org.

DOI:10.1523/JNEUROSCI.3968-12.2013

Copyright $\odot 2013$ the authors $\quad 0270-6474 / 13 / 332365-11 \$ 15.00 / 0$
}

The skills that rehabilitation attempts to restore normally depend on plasticity throughout the CNS, from the cortex to the spinal cord (Drew et al., 2002; Nielsen, 2002; Hultborn and Nielsen, 2007; Wolpaw, 2010; Rossignol and Frigon, 2011). Moreover, the location and nature of the damage that impairs performance differ widely from individual to individual, as well as from disorder to disorder. As a result, the plastic changes needed to restore a particular skill (e.g., locomotion) are also likely to differ widely across individuals. Thus, new therapeutic methods that can induce plasticity in particular CNS pathways, and can thereby target each individual's particular deficits, might significantly increase the effectiveness of rehabilitation.

In both animals and humans, operant conditioning protocols can modify specific spinal reflex pathways (Wolpaw et al., 1983; Wolpaw and O'Keefe, 1984; Wolpaw, 1987; Chen and Wolpaw, 1995; Wolf and Segal, 1996; Carp et al., 2006b; Chen et al., 2006a; Thompson et al., 2009). Because these spinal pathways participate in important skills such as locomotion, conditioning protocols might be used to reduce the functional deficits produced by spinal cord injuries, strokes, and other disorders. An initial animal study supports this hypothesis. In rats in which a lateralized spinal cord injury (SCI) had produced a gait asymmetry, appropriate conditioning of the soleus $\mathrm{H}$-reflex on the injured side eliminated the asymmetry and restored more normal locomotion (Chen et al., 2006b).

The present study is, to our knowledge, the first effort to use spinal reflex conditioning to improve function in people with SCI. It focuses on people in whom a chronic incomplete SCI has 
Table 1. Profiles of DC and UC subjects

\begin{tabular}{lllllll}
\hline Group & $\begin{array}{l}\text { Age } \\
\text { (years) }\end{array}$ & Gender & Cause & $\begin{array}{l}\text { SCl } \\
\text { level }\end{array}$ & AIS & $\begin{array}{l}\text { Years } \\
\text { post-SCI }\end{array}$ \\
\hline DC & 61 & M & NT & C7 & D & 10 \\
& 68 & M & T & C3 & D & 2.5 \\
& 37 & M & T & T6 & D & 0.7 \\
& 39 & M & T & T11 & D & 1 \\
& 30 & F & T & C & D & 7 \\
& 34 & M & T & C2 & C & 1.5 \\
& 51 & M & T & C & D & 0.8 \\
& 65 & F & NT & T4 & D & 49 \\
& 48 & F & NT & T7 & D & 5 \\
UC & 53 & M & T & C5 & D & 3 \\
& 28 & M & T & C7 & C & 5.5 \\
& 67 & F & NT & T4 & D & 50 \\
& 48 & M & T & C3 & D & 0.8 \\
\hline
\end{tabular}

M, Male; F, female; T, trauma; NT, non-trauma; SCl level, the highest spinal cord level that was damaged; AIS, American Spinal Injury Association Impairment Scale.

produced a spastic gait disorder characterized by hyperreflexia and abnormal reflex modulation in ankle extensor muscles (Dietz and Sinkjaer, 2007; Nielsen et al., 2007). By downconditioning the soleus H-reflex, the study sought to reduce these abnormalities, and to thereby improve the speed and symmetry of locomotion. The results are clear and encouraging. They suggest that operant conditioning protocols could provide a valuable new approach to restoring useful function after spinal cord injuries or in other neuromuscular disorders.

\section{Materials and Methods}

Subjects. The study participants were 13 ambulatory subjects ( 9 men and 4 women; age range, $28-68$ years; mean \pm SD age, $48.4 \pm 13.9$ years) (Table 1) who had suffered an SCI 8 months to 50 years earlier that had impaired locomotion. All subjects gave informed consent for the study, which was reviewed and approved by the Institutional Review Board of Helen Hayes Hospital. A physiatrist (F.R.P.) determined each prospective subject's eligibility for the study. The inclusion criteria were as follows: (1) a stable SCI-related motor deficit (>6 months after lesion); (2) ability to ambulate at least $10 \mathrm{~m}$ either with or without an assistive device (e.g., cane, crutches, or walker); (3) signs of spasticity (i.e., exaggerated H-reflexes, increased muscle tone, score $\geq 1$ on Modified Ashworth scale) and weak ankle dorsiflexion (i.e., manual dorsiflexor muscle strength at ankle $<5$ ) unilaterally or bilaterally; (4) a reasonable expectation that current medications would not change over the period of the study (e.g., an anti-spasticity medication such as baclofen, diazepam, or dantrolene); and (5) medical clearance to participate. The exclusion criteria were as follows: (1) a lower motoneuron injury; (2) a known cardiac condition; (3) another medically unstable condition; (4) cognitive impairment; and/or (5) daily use of functional electrical stimulation to counteract foot drop. In the subjects who exhibited bilateral motor impairments, the soleus H-reflex of the more impaired leg was studied.

The subjects were randomly assigned (at a 2:1 ratio) to the downconditioning (DC) group ( 6 men and 3 women; age range, $30-68$ years; mean \pm SD age, $48.2 \pm 14.0$ years; subjects $1-9$ in Table 1 ) or the unconditioned (UC) group (3 men and 1 woman; age range, 28-67 years; mean \pm SD age, $48.8 \pm 15.7$ years; subjects $10-13$ in Table 1$)$. The primary purpose of the UC group was to establish that $\mathrm{H}$-reflex decrease was specific to the down-conditioning protocol.

Operant conditioning of the soleus H-reflex: overview. The operant conditioning protocol for the human soleus H-reflex was originally developed in a study of neurologically normal subjects and is described in detail in Thompson et al. (2009). It is summarized here, with several minor modifications noted.

Figure 1 summarizes the protocol. After one to three preliminary sessions in which appropriate background EMG and M-wave criteria were defined, each subject completed 6 baseline sessions and 30 control (UC subjects) or conditioning (DC subjects) sessions at a rate of 3 sessions per week. Each session lasted $\sim 1 \mathrm{~h}$ and occurred within the same $2 \mathrm{~h}$ time window (to prevent the normal diurnal variation in reflex size from affecting the results (Wolpaw and Seegal, 1982; Chen and Wolpaw, 1994; Carp et al., 2006a; Lagerquist et al., 2006). As Figure $1 B$ shows, in the 6 baseline sessions of all subjects, and in the 30 control and 2 follow-up sessions of the UC subjects, 225 control H-reflexes (in three 75-trial blocks) were elicited during standing. In these 225 control trials, there was no feedback to the subject regarding $\mathrm{H}$-reflex size. In contrast, in the 30 conditioning and 2 follow-up sessions of the DC subjects, 20 control H-reflexes were elicited, and then 225 conditioned H-reflexes (in three 75 -trial blocks) were elicited. In these 225 conditioning trials, the subject was asked to decrease the H-reflex and was given immediate visual feedback after each stimulus (see below) to indicate whether the resulting H-reflex was smaller than a criterion value. Background EMG and M-wave size were kept stable throughout data collection.

Electrical stimulation and EMG recording. At the beginning of each session, EMG recording and stimulating electrodes were placed over the leg. EMG activity from soleus and its antagonist tibialis anterior (TA) was recorded with surface self-adhesive $\mathrm{Ag}$ - $\mathrm{AgCl}$ electrodes $(2.2 \times 3.5 \mathrm{~cm}$, Vermed), amplified, bandpass filtered $(10-1000 \mathrm{~Hz})$, digitized $(5000$ $\mathrm{Hz}$ ), and stored. To elicit the H-reflex, the tibial nerve was stimulated in the popliteal fossa, using surface $\mathrm{Ag}$ - $\mathrm{AgCl}$ electrodes $(2.2 \times 2.2 \mathrm{~cm}$ for the cathode and $2.2 \times 3.5 \mathrm{~cm}$ for the anode; Vermed) and a Grass S88 stimulator (with a CCU1 constant current unit and an SIU5 stimulus isolation unit; Astro-Med). The stimulating electrode pair was placed so as to minimize the H-reflex threshold and to avoid stimulating other nerves. This placement was accomplished by monitoring the EMG of soleus and TA and palpating other lower-leg muscles, such as the peroneal muscle group. To avoid session-to-session variability in electrode placement, their positions were mapped in relation to landmarks on the skin (e.g., scars or moles). The same individuals (A.K.T. assisted by Briana M. Abel) placed the electrodes and conducted every session for every subject.

The soleus H-reflex was elicited by a $1 \mathrm{~ms}$ square-pulse stimulus while the subject maintained a natural standing posture with hands resting on a horizontal bar at waist height and with stable levels of soleus and TA background EMG activity. The stimulus occurred after the subject had maintained rectified soleus and TA EMG activity within specified ranges for at least $2 \mathrm{~s}$. Typically, the soleus range was $10-20 \%$ of a maximum voluntary contraction, and the TA range was $0-7 \mu \mathrm{V}$ (i.e., resting level). The minimum interstimulus interval was $5 \mathrm{~s}$.

Session protocol. At the beginning of each session, an H-reflex/M-wave (H-M) recruitment curve was obtained. All H-reflex and M-wave measurements were reported in absolute values (i.e., equivalent to rectified EMG). Stimulus intensity was varied in increments of $1.25-2.50 \mathrm{~mA}$ from below soleus $\mathrm{H}$-reflex threshold, to the maximum $\mathrm{H}$-reflex $\left(\mathrm{H}_{\max }\right)$, to an intensity just above that needed to elicit the maximum $\mathrm{M}$-wave $\left(\mathrm{M}_{\max }\right)$ (Kido et al., 2004b; Makihara et al., 2012). Approximately 10 different intensities were used to obtain each recruitment curve. At each intensity, four EMG responses were averaged to measure the H-reflex and $\mathrm{M}$-wave. The stimulus amplitude used for the subsequent $\mathrm{H}$-reflex trials fell on the rising phase of the H-reflex recruitment curve and typically produced an $\mathrm{M}$-wave just above threshold. In each subject, this $\mathrm{M}$-wave size was maintained for the H-reflex trials of all the sessions.

In the baseline and control sessions (Fig. 1B), the H-M recruitment curve was followed by three 75-trial blocks of control trials, in which the subject was not asked to change the H-reflex and was not given visual feedback as to H-reflex size (see Visual feedback). In the conditioning sessions (Fig. $1 B$ ), the H-M recruitment curve was followed by a 20 -trial block of control trials identical to those of the baseline or control sessions; and this block was followed by three 75 -trial blocks of conditioning trials, in which the subject was asked to decrease H-reflex size and was provided with immediate visual feedback that indicated his or her success in doing so (see below).

Visual feedback. The visual feedback screens for control and conditioning trials have been described in detail previously (Thompson et al., 2009) and are illustrated in Figure 1C. Briefly, the screen could present two graphs, one for soleus background EMG activity and one for H-reflex size. In control trials, only the background EMG graph was shown: if the 


\section{A Session schedule}

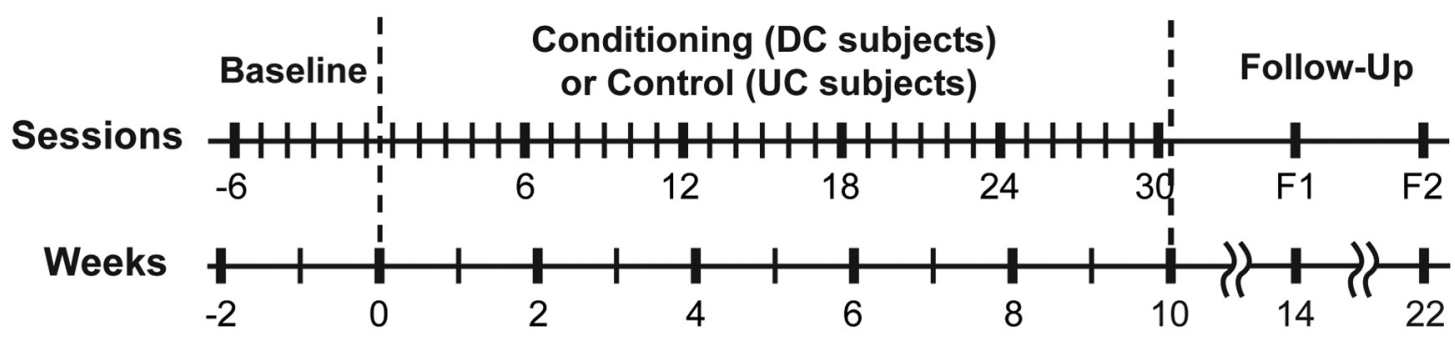

\section{B Session composition}

\begin{tabular}{|c|c|c|}
\hline & $\begin{array}{l}\text { Unconditioned (UC) } \\
\text { Subjects }\end{array}$ & $\begin{array}{c}\text { Down-Conditioning (DC) } \\
\text { Subjects }\end{array}$ \\
\hline Baseline Sessions & \multicolumn{2}{|c|}{$\begin{array}{c}\text { H-M recruitment curve } \\
225 \text { Control Trials (three } 75 \text {-trial blocks) }\end{array}$} \\
\hline $\begin{array}{c}\text { Control / Conditioning } \\
\text { Sessions } \\
\& \\
\text { Follow-Up Sessions }\end{array}$ & $\begin{array}{c}\text { H-M recruitment curve } \\
225 \text { Control Trials } \\
\text { (in three } 75 \text {-trial blocks) }\end{array}$ & $\begin{array}{l}\text { H-M recruitment curve } \\
20 \text { Control Trials } \\
225 \text { Conditioning Trials } \\
\text { (in three } 75 \text {-trial blocks) }\end{array}$ \\
\hline
\end{tabular}

C Visual feedback screens

Control trials

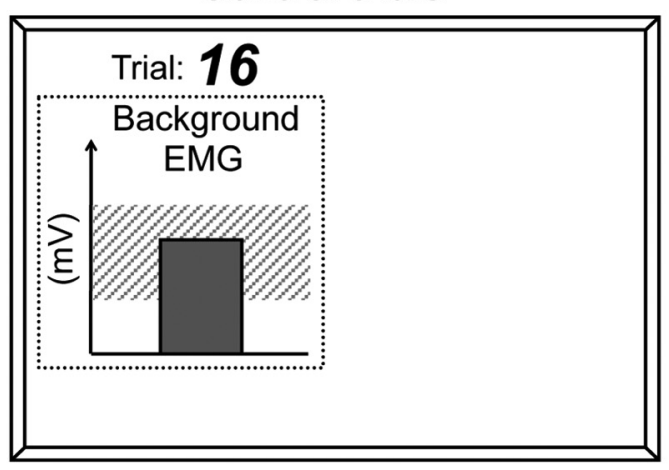

Conditioning trials

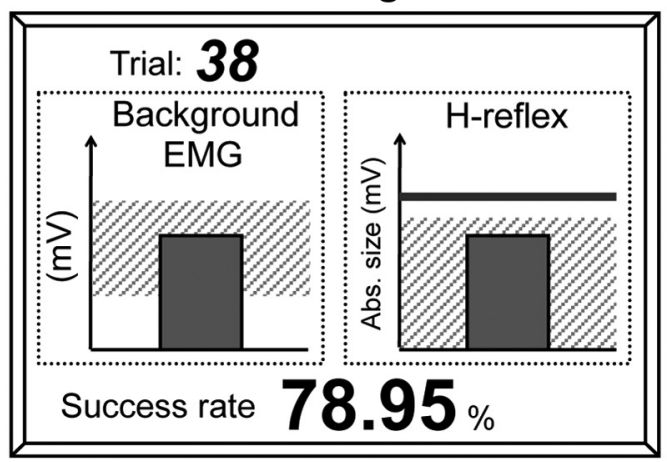

Figure 1. $\boldsymbol{A}$, Session schedule. Six baseline sessions were followed by 30 conditioning (DC subjects) or control (UC subjects) sessions, and then by two follow-up sessions. $\boldsymbol{B}$, Composition of baseline, control, conditioning, and follow-up sessions. C, Visual feedback screens for control and conditioning trials (see text for full description).

subject kept the height of the vertical bar (i.e., soleus background EMG activity level, in absolute value) in the specified range for $2 \mathrm{~s}$, and at least $5 \mathrm{~s}$ had passed since the last stimulus, a stimulus pulse elicited the $\mathrm{H}$-reflex and $\mathrm{M}$-wave. In conditioning trials, the background EMG graph was shown, and, in addition, the H-reflex size graph was shown. This graph constantly showed a heavy horizontal line indicating the subject's average H-reflex size for the six baseline sessions and a shaded area that indicated the H-reflex size range that satisfied the current down-conditioning criterion value. Two hundred milliseconds after the stimulus, a vertical bar reflecting H-reflex size appeared. The bar was green (indicating success) when the H-reflex fell within the shaded area (i.e., was below the criterion value), and the bar was red (indicating failure) when the H-reflex size was not below the criterion value. In addition, the current success rate (i.e., the percentage of the trials of the current 75-trial block that were successful) was shown below the graph and was updated after each trial. Thus, for each control trial, the visual feedback simply helped the subject maintain the required prestimulus background EMG activity. In contrast, for each conditioning trial, the visual feedback also informed subjects as to whether they had succeeded in producing an $\mathrm{H}$-reflex small enough to satisfy the size criterion, and it showed the success rate for the current block of trials.

In each conditioning session, the criterion value for the first block of 75 conditioning trials was based on the immediately preceding block of 20 control trials, and the criterion values for the second and third blocks of conditioning trials were based on the $\mathrm{H}$-reflexes of the immediately preceding block of 75 conditioning trials. The criterion was selected so that if $\mathrm{H}$-reflex values for the new block were similar to those for the previous block, $50-60 \%$ of the trials would be successful (Chen and Wolpaw, 1995). For each block, the subject earned a modest extra monetary reward when the success rate exceeded $50 \%$ (for full details, see Thompson et al., 2009).

Analysis of conditioned and control H-reflexes. For each session of each subject, we determined the average H-reflex size for the 225 trials of the three 75-trial blocks (Fig. 1B). This value is called the conditioned $\mathrm{H}$-reflex size (regardless of whether the session is from a DC subject or a UC subject). In addition, for each session of each subject we determined the average H-reflex size for 20 control trials. This value is called the control $\mathrm{H}$-reflex size. For the 6 baseline sessions of all subjects and the 30 control sessions of the UC subjects, these 20 control trials were the first 20 
trials of the first 75 -trial block. For the 30 conditioning sessions and the follow-up sessions of the DC subjects, these 20 control trials were elicited before the three 75 -trial blocks of conditioning trials, as indicated in Figure $1 B$. H-reflex size was defined as the average absolute value of soleus EMG (i.e., equivalent to rectified EMG) in the H-reflex interval (typically $30-45 \mathrm{~ms}$ after the stimulus) minus the average absolute value of soleus background EMG.

To determine for each subject whether the conditioned H-reflex size changed significantly over the 30 conditioning or control sessions, the average $\mathrm{H}$-reflexes for the 225 trials of the three 75 -trial blocks of the final six sessions (i.e., sessions 25-30) were compared with the average $\mathrm{H}$-reflexes for the 225 trials of the three 75 -trial blocks of the six baseline sessions by unpaired $t$ test (two tailed). To determine for each subject the final conditioned $\mathrm{H}$-reflex size, the average $\mathrm{H}$-reflexes for the 225 trials of the three 75-trial blocks of the final three sessions (i.e., sessions 28-30) were averaged, and the result was expressed as the percentage of the average H-reflex for the 225 trials of the three 75 -trial blocks of the six baseline sessions. (Thus, a value of $100 \%$ indicated no change.) To determine for each subject the final control H-reflex size, the average H-reflexes for the 20 control trials of sessions 28-30 were averaged, and the result was expressed as the percentage of the average H-reflex for the 20 control trials of the six baseline sessions.

To assess the stability of soleus $\mathrm{M}_{\max }$, soleus $\mathrm{M}$-wave size in control and conditioning trials, and soleus and TA background EMG levels, a repeated-measures ANOVA was applied to the mean values across successive six-session blocks beginning with the six baseline sessions. Soleus $\mathrm{M}_{\text {max }}$, soleus $\mathrm{M}$-wave size during $\mathrm{H}$-reflex elicitation, and soleus and TA background EMG levels remained stable across all the sessions in both the DC and UC groups ( $p>0.33$ for all of these measures in both groups, one-way repeated-measures ANOVA). These results confirmed the stability of EMG recording and nerve stimulation conditions in this study, and thus supported the validity of the methodology.

Assessment of locomotion. Locomotion was assessed before and after the 30 conditioning or control sessions. These assessments occurred on non-session days. First, the subject was asked to walk $10 \mathrm{~m}$ overground at a comfortable speed three times, and the average walking time was determined.

Then, locomotor symmetry, EMG activity, and H-reflex modulation were measured. For these measurements during locomotion, any subject who wore an ankle foot orthosis was asked to remove it. Surface EMG was recorded from the soleus, TA, vastus lateralis (VL), and biceps femoris (BF) muscles of both legs. Footswitch cells inserted between the subject's shoe and foot detected foot contact (typically, heel or toe contact). For locomotor H-reflex measurement, single $1 \mathrm{~ms}$ square-pulse stimuli were delivered at different points in the step cycle to evaluate phase-dependent H-reflex modulation (Capaday and Stein, 1986; Stein and Capaday, 1988; Ethier et al., 2003; Kido et al., 2004b). The stimulus interval was set to be long enough to have at least one full unstimulated step cycle between successive stimuli.

Those subjects who were able to walk on a treadmill for several minutes with a consistent stepping rhythm did so twice at a comfortable speed: once without $\mathrm{H}$-reflex elicitation and once while tibial nerve stimulation elicited the soleus H-reflex. Subjects who were not able to walk on the treadmill repeated $10 \mathrm{~m}$ overground walking without stimulation until at least 50 steps were obtained. They then repeated $10 \mathrm{~m}$ overground walking with H-reflex elicitation until at least 50 stimulated steps were obtained. During these measurements, the subjects took sitting breaks as often as needed. The data were assessed as described below, and the locomotor measurements obtained before and after the 30 conditioning or control sessions were compared.

For analysis of locomotor EMG activity, the complete step cycle was divided into 12 bins of equal duration (Kido et al., 2004a; Makihara et al., 2012). For the muscles of the conditioned leg, the step cycle went from the conditioned leg's foot contact ( $\mathrm{cFC}$ ) to the next cFC; for the muscles of the contralateral (i.e., nonconditioned) leg, the step cycle went from the nonconditioned leg's foot contact (nFC) to the next nFC. For each muscle of each subject, the average rectified EMG amplitude in each of the 12 bins was determined and expressed as a percentage of the amplitude in the bin with the highest amplitude. The degree to which each muscle's activity was modulated during locomotion was determined by calculating its modulation index $(\mathrm{MI})$ in percent as: $100 \times[$ (highest bin amplitude - lowest bin amplitude)/highest bin amplitude] (Zehr and Kido, 2001; Zehr and Loadman, 2012). Thus, an MI of 0\% indicated that a muscle did not modulate its activity at all over the step cycle.

To assess gait quality, we examined step-cycle symmetry (i.e., the ratio of the time between the $\mathrm{nFC}$ and the $\mathrm{cFC}$ to the time between $\mathrm{cFC}$ and $\mathrm{nFC})$. A ratio of 1 indicates a symmetrical gait.

Spontaneous subject comments. Over the 3 months of the study, the subjects were not asked about the current state of their motor function or whether their disabilities had changed in any way. Nevertheless, many volunteered comments when they came in for sessions. We kept a record of these spontaneous comments and when they were first made. They fall into distinct categories and tell a clear story both in their nature and in their timing. Thus, they are presented as a unique and important component of the results.

\section{Results}

All 13 subjects completed the 6 baseline sessions and 30 conditioning or control sessions. In each subject, soleus $\mathrm{M}_{\text {max }}$, soleus $\mathrm{M}$-wave size in control and conditioning trials, and soleus and TA background EMG levels remained stable across all the sessions. The DC and UC groups did not differ significantly in soleus and TA background EMG levels ( $p=0.90$ and 0.81 by two-way repeated-measures ANOVA, respectively), M-wave sizes ( $p=$ $0.71)$, or baseline H-reflex sizes $(p=0.11)$.

The results comprise three categories of data: conditioned and control H-reflex sizes over the course of the sessions; locomotor speed, symmetry, EMG activity, and H-reflex modulation before and after the 30 conditioning or control sessions; and the spontaneous comments of the subjects over the course of the sessions. These three datasets are described here.

\section{H-reflex size}

Figure $2 A$ shows the final conditioned $H$-reflex sizes of the DC subjects, the UC subjects, and, for comparison, the normal DC subjects of Thompson et al. (2009). The filled triangles represent the DC subjects in whom down-conditioning was successful (i.e., the average conditioned H-reflexes for conditioning sessions 25-30 were significantly less than those for the 6 baseline sessions). In the other DC subjects (open triangles), the H-reflex did not change significantly. The success rate for the subjects with SCI (i.e., 6/9 or 67\%) is slightly, but not significantly, less than that for neurologically normal subjects (i.e., $8 / 9$ or $89 \%$ ) (Thompson et al., 2009) or for normal monkeys, rats, and mice (i.e., 75-80\%) (Wolpaw et al., 1983; Wolpaw, 1987; Chen and Wolpaw, 1995; Carp et al., 2006b). In contrast, the conditioned H-reflex did not decrease significantly in any of the UC subjects; and the DC and UC groups differed significantly in final H-reflex size ( $p=0.025$ by unpaired $t$ test). Thus, H-reflex decrease was specific to the DC group. Indeed, it should be noted that the UC group as a whole showed a slight but significant increase in the conditioned H-reflex [to (mean \pm SE) $116 \pm 7 \%$ of baseline; $p=$ 0.05 by paired $t$ test]. This may have been a nonspecific effect of continued exposure over 30 sessions to the baseline protocol of standing, providing soleus background EMG, and having the H-reflex elicited.

As noted in our previous study of H-reflex conditioning in normal subjects (Thompson et al., 2009), successful DC subjects reported that, in the first four to five conditioning sessions, they tried different strategies for decreasing the H-reflex, identified an effective strategy, and then used it in subsequent conditioning trials. Their reported techniques were comparable to those of normal subjects (Table 2 in Thompson et al., 2009) (e.g., meditation, anticipating stimulus occurrence). 

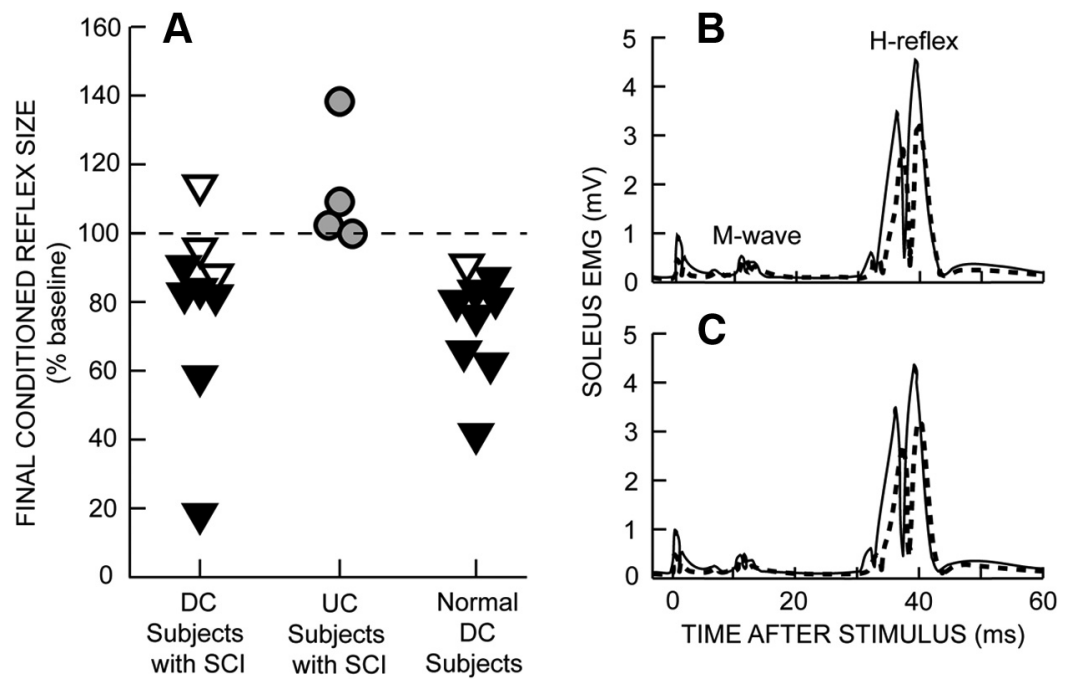

Figure 2. $\quad A$, Final conditioned H-reflex sizes (i.e., average for the last three conditioning sessions) for individual $D C$ and UC subjects. The data for normal DC subjects are from Thompson et al. (2009). The filled triangles represent the DC subjects whose conditioned $\mathrm{H}$-reflexes for the last six conditioning sessions were significantly less than their $\mathrm{H}$-reflexes for the six baseline sessions. The open triangles represent the DC subjects in whom the H-reflex did not decrease significantly. (The lowest open triangle failed to reach statistical significance due to high intersession variability.) $\boldsymbol{B}, \boldsymbol{C}$, Average conditioned $(\boldsymbol{B})$ and control (C) H-reflexes for a baseline session (solid line) and the last conditioning session (dashed line) from a DC subject with $\mathrm{SCl}$ in whom the H-reflex decreased significantly. Both conditioned and control H-reflexes are smaller after 30 conditioning sessions. As summarized in Figure $3, A$ and $B$, the decrease in the control $\mathrm{H}$-reflex is almost as great as that in the conditioned $\mathrm{H}$-reflex. Background EMG and $M$-wave values do not change. A small stimulus artifact is present.

Figure 2, $B$ and $C$, shows H-reflexes from a successful DC subject during the baseline period (solid) and at the end of the 30 conditioning sessions (dashed). Figure $2 B$ illustrates the change in the conditioned H-reflex (i.e., the H-reflex for the three 75trial blocks in which the subject was asked to decrease the $\mathrm{H}$-reflex and was provided with immediate feedback as to whether the reflex satisfied the size criterion). Figure $2 C$ illustrates the change in the control H-reflex (i.e., the H-reflex for the first 20 trials of each conditioning session in which the subject was not asked to decrease the H-reflex and was not provided with feedback as to reflex size). Both the conditioned and control $\mathrm{H}$-reflexes are smaller after down-conditioning. Background soleus EMG level and M-wave size do not change.

Figure 3 shows the average courses of H-reflex changes for subjects with SCI (Fig. 3A-C, from this study) and for normal subjects (Fig. 3D-F, from Thompson et al., 2009) in whom down-conditioning was successful. Figure $3, A$ and $D$, shows the conditioned $H$-reflex change. Figure $3, B$ and $E$, shows the control H-reflex change. Finally, Figure 3, $C$ and $F$, shows the change in the within-session difference between the conditioned and control H-reflexes. This difference represents task-dependent adaptation; that is, the decrease that the subjects were able to produce immediately when they were asked to decrease the H-reflex.

These courses of change are noteworthy in several respects. First, the final average value of the conditioned H-reflex (i.e., the average of the last three conditioning sessions) in the subjects with SCI is identical to that in normal subjects [i.e., (mean $\pm \mathrm{SE}$ ) $69 \pm 11 \%$ and $69 \pm 6 \%$ of baseline, respectively]. Second, the final value of the control H-reflex in the subjects with SCI is significantly smaller than in normal subjects (i.e., $76 \pm 9 \%$ of baseline vs $84 \pm 6 \%$, respectively; $p=0.01$, two-tailed $t$ test). Thus, the subjects with SCI decreased the control H-reflex more than normal subjects. Third, like normal subjects, the subjects with SCI display an appropriate task-dependent adaptation (i.e., a session's average conditioned H-reflex is smaller than its aver-

\section{Walking speed}

age control H-reflex) that begins after four conditioning sessions and remains about the same thenceforth. However, this task-dependent adaptation is significantly less in the subjects with SCI than in normal subjects (i.e., averages of $-7 \pm$ $3 \%$ and $-15 \pm 6 \%$, respectively; $p=0.01$, two-tailed $t$ test). The greater decrease in the control H-reflex in the subjects with SCI combines with their lesser taskdependent adaptation to yield a decrease in the conditioned H-reflex that is identical to that found in normal subjects.

Four of the successful DC subjects completed follow-up sessions 1 month and 3 months after the conditioning sessions ended. At both 1 month and 3 months, the conditioned H-reflex remained reduced in every subject, averaging (mean \pm SE) $65 \pm 10 \%$ and $58 \pm 10 \%$ of baseline values, respectively. One DC subject also completed a 6 month follow-up session. The conditioned H-reflex was $26 \%$ of baseline, comparable to the value of $18 \%$ for the final three conditioning sessions.

In the successful DC subjects, the $\mathrm{H}_{\text {max }}$ measured at the beginning of each session also decreased with down-conditioning, paralleling the changes in the control H-reflex [final value (mean $\pm \mathrm{SE}$ ), $84 \pm 5 \%$ of baseline; $p<0.001$, paired $t$ test]. This decrease paralleled the decrease in the control H-reflex and was consistent with the effects of H-reflex conditioning on the H-reflex recruitment curve (Thompson et al., 2012). In contrast, $\mathrm{H}_{\max }$ did not change significantly in the seven subjects in whom the H-reflex did not decrease.

\section{Locomotor speed, symmetry, EMG activity, and H- reflex modulation}

Over the 30 conditioning or control sessions, the subjects' $10 \mathrm{~m}$ walking speeds increased by $0-123 \%$. The increase was substantial and significant in the six DC subjects in whom the H-reflex decreased $[($ mean $\pm \mathrm{SE})+59 \pm 19 \% ; p=0.03$, paired $t$ test $]$. Furthermore, the two subjects who increased their walking speeds most also decreased their dependence on an assistive device in their daily lives: one switched from a walker to crutches, and the other switched from a walker to a cane. In contrast, in the seven subjects in whom the H-reflex did not decrease, walking speed increased less and not significantly $(+25 \pm 13 \%$; $p=0.10)$. In none of these subjects did dependence on an assistive device change. Figure $4 A$ summarizes these results.

\section{Locomotor symmetry}

To assess gait quality, we examined step-cycle symmetry (i.e., the ratio of the time between the $\mathrm{nFC}$ and the $\mathrm{CFC}$ to the time between $\mathrm{cFC}$ and $\mathrm{nFC}$ ). A ratio of 1 indicates a symmetrical gait. During the baseline period, the ratio was always $>1$ because foot drop and/or spasticity prolonged the swing phase of the conditioned leg (which was the more impaired leg) and/or because spasticity and the resulting instability in the conditioned leg shortened its stance (i.e., weight-bearing) phase. After the 30 conditioning or control sessions, this ratio decreased in every subject 
in whom the H-reflex decreased, becoming closer to 1 ( $p=0.05$, paired $t$ test). In contrast, the ratio increased in every subject in whom the H-reflex did not decrease $(p=0.02)$. Figure $4 B$ summarizes these results. Thus, the successful DC subjects walked faster and more symmetrically; while the subjects in whom the H-reflex did not decrease walked slightly but not significantly faster and walked less symmetrically.

Figure $4 \mathrm{C}$ shows the $\mathrm{nFC}-\mathrm{cFC}$ and $\mathrm{cF}-$ C-nFC time intervals in one DC subject before and after successful conditioning. Before conditioning, the $\mathrm{nFC}-\mathrm{cFC}$ time interval was longer than the $\mathrm{FC}-\mathrm{nFC}$ interval. After conditioning, the two intervals were equal, indicating that locomotion had become more symmetrical.

\section{Locomotor EMG activity}

To further assess changes in walking, locomotor EMG activity was recorded from the soleus, TA, VL, and BF muscles of both legs before and after the 30 conditioning or control sessions, and each muscle's MI was determined as described in the Materials and Methods.

MI values varied widely across subjects and across the eight muscles of each subject, with many abnormally low values (i.e., $>2$ SDs below the average for 12 normal subjects; unpublished data). In the DC subjects in whom the H-reflex decreased, the average MI rose significantly [from (mean \pm SD) $74 \pm 17 \%$ to $80 \pm$ $11 \% ; p=0.005$, paired $t$ test]. This improvement was bilateral; it was not limited to the muscles of the conditioned leg. Thus, successful H-reflex down-conditioning was associated with a significant increase in the degree to which ankle and knee flexor and extensor muscles of both legs modulated their activity in synchrony with the step cycle. In the seven subjects in whom the H-reflex did not decrease, the average MI did not change ( $84 \pm 8 \%$ before and $84 \pm 10 \%$ after; $p=0.25$ ). [Although the average initial MI was higher in this group, it was still below normal (i.e., $89 \%$; unpublished data]. Thus, this lack of increase cannot be attributed simply to a ceiling effect.)

Figure 5 shows modulation over the step cycle in the muscles of both legs for one DC subject before and after successful down-conditioning. After conditioning, soleus activity is increased and TA activity is decreased during midto-late stance in both legs. These bilateral improvements in the modulation of muscle activity controlling movement about the ankle joint probably resulted in more effective weight bearing and push off, and thereby contributed to this subject's increased walking speed (from 0.59 to $0.80 \mathrm{~m} / \mathrm{s}$ ). Locomotor EMG modulation also increased in other muscles.

\section{Subjects with SCI}

\section{Normal Subjects}
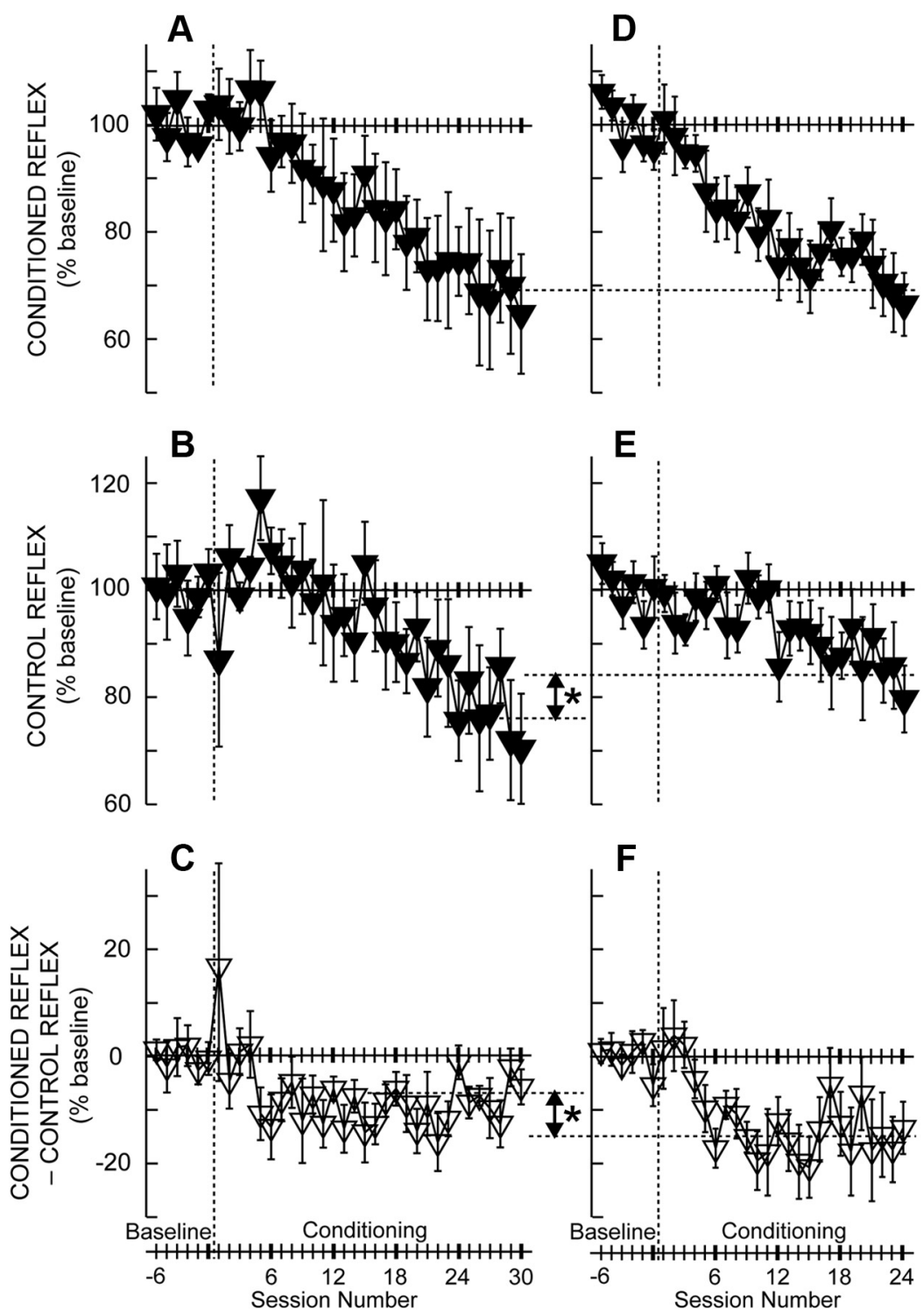

Figure 3. $A-F$, Average ( $\pm \mathrm{SE}) \mathrm{H}$-reflex values for baseline and conditioning sessions for $\mathrm{DC}$ subjects with $\mathrm{SCl}(\boldsymbol{A}-\mathrm{C}, \mathrm{N}=6$; this study) and for normal subjects ( $\boldsymbol{D}-\boldsymbol{F}, N=8$; study by Thompson et al., 2009) in whom the H-reflex decreased significantly. $\boldsymbol{A}, \boldsymbol{D}$, Average conditioned H-reflex size. $\boldsymbol{B}, \boldsymbol{E}$, Average control H-reflex size. $\boldsymbol{C}, \boldsymbol{F}$, Average of conditioned H-reflex size minus control H-reflex size (i.e., task-dependent adaptation; see Results) (for details, see Thompson et al. (2009)). In the subjects with SCI, the conditioned $\mathrm{H}$-reflex decreases to $69 \%$ of the baseline value over 30 conditioning sessions $(\boldsymbol{A})$. This decrease consists of a relatively small task-dependent adaptation $(-7 \%, \boldsymbol{C})$ and a relatively large across-session control reflex decrease $(-24 \%, \boldsymbol{B})$. In the subjects without disability (Thompson et al., 2009), the conditioned H-reflex also decreases to $69 \%$ of the baseline value over 24 conditioning sessions (D). This decrease consists of a relatively large task-dependent adaptation $(-15 \%, \boldsymbol{F})$ and a relatively small across-session control reflex decrease $(-16 \%, \boldsymbol{E})$. The asterisks between $\boldsymbol{B}$ and $\boldsymbol{E}$ and between $\boldsymbol{C}$ and $\boldsymbol{F}$ indicate significant differences $(p<0.01)$ between subjects with $\mathrm{SCl}$ and normal subjects in final control $\mathrm{H}$-reflex value and in task-dependent adaptation, respectively. Task-dependent adaptation is greater in the normal subjects, while change in the control H-reflex is greater in the subjects with SCl.

\section{$H$-reflex modulation during locomotion}

In addition to recording EMG activity during undisturbed locomotion, we also elicited soleus H-reflexes during locomotion. As described above for locomotor EMG analysis, the step cycle was divided into 12 bins of equal duration, and average $\mathrm{H}$-reflex size for each bin was determined. The average of these 12 values defined the average locomotor H-reflex.

In the successful DC subjects, the average locomotor H-reflex also decreased [to (mean \pm SE) $59 \pm 17 \%$ of baseline value; $p=$ 
A

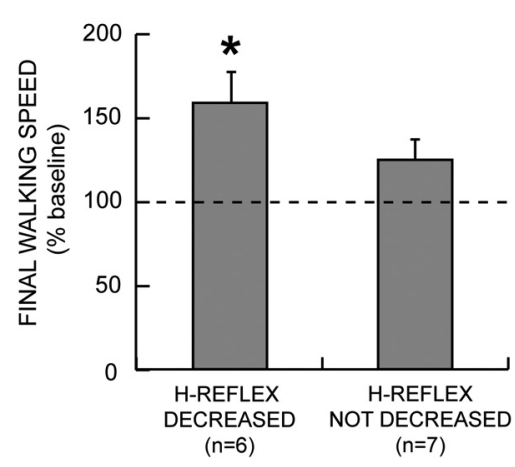

B

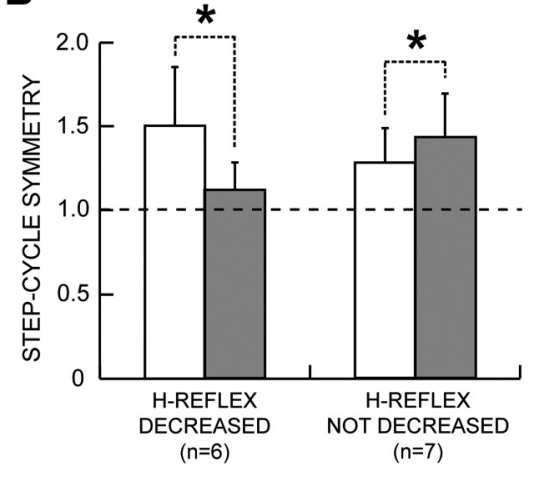

C

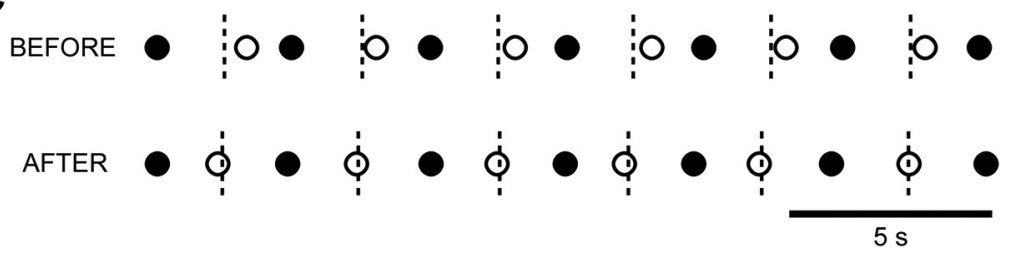

Figure 4. $\quad A$, Average ( \pm SE) $10 \mathrm{~m}$ walking speeds after 30 conditioning or control sessions (in percentage of baseline speed) for subjects in whom the H-reflex did or did not decrease significantly. $\boldsymbol{B}$, Step-cycle symmetry before (open bars) and after (shaded bars) 30 conditioning or control sessions for subjects in whom the H-reflex did or did not decrease significantly. Symmetry is measured as the ratio of the time between the $\mathrm{nFC}$ and the CFC (or simply stimulated, in the case of UC subjects) to the time between $\mathrm{CFC}$ and $\mathrm{nFC}$. A ratio of 1 indicates a symmetrical gait. Initially, the ratio is $>1$. After the 30 conditioning or control sessions, the ratio has decreased toward 1 in the subjects in whom the $\mathrm{H}$-reflex decreased, while it has increased in the subjects in whom the H-reflex did not decrease. C, Successive step cycles before and after conditioning from a subject in whom the H-reflex decreased. Each $\mathrm{nFC}(\mathbf{O})$ and $\mathrm{cFC}(\bigcirc)$ are shown. The short vertical dashed lines mark the midpoints between nFCs (i.e., the midpoints of the step cycle), which is when cFC should occur. Before H-reflex down-conditioning, CFC occurs too late; after successful down-conditioning, it occurs on time.

0.04 by paired $t$ test]. Thus, in humans as in rats (Chen et al., 2005, 2006b), an H-reflex decrease produced by the conditioning protocol was also evident during locomotion. The decreased average locomotor H-reflex reflected the combination of an overall decrease throughout the step cycle and a decrease concentrated in the swing phase, the period when the locomotor $\mathrm{H}$-reflex is small in normal subjects. In contrast, in the subjects in whom the $\mathrm{H}$-reflex did not decrease, the average locomotor $\mathrm{H}$-reflex showed an insignificant increase (to $125 \pm 17 \%$ of baseline value; $p=0.15)$.

Figure 6 illustrates the decrease in the locomotor H-reflex of one successful DC subject. It is markedly reduced throughout the step cycle. In addition, its modulation across the step cycle has become more normal: the reflex is smallest during the swing phase. Locomotor soleus EMG has also become more normal, with much less inappropriate activity during the swing phase.

\section{Spontaneous subject comments}

Although subjects were not questioned about the current state of their disabilities during the study, many volunteered spontaneous comments. The 13 subjects made a total of 30 positive comments of 10 different kinds and no negative comments.

Figure 7 lists these 10 different kinds of positive comments and indicates when during the study they were first made by the DC subjects in whom the H-reflex decreased significantly and by the subjects in whom the H-reflex did not decrease. The contrast is striking: 25 of the 30 positive comments were made by the six DC subjects in whom down-conditioning was successful, and only 5 positive comments by the other seven subjects $(p=$
0.0027, Mann-Whitney $U$ test). All six successful DC subjects reported walking faster and farther, and one to three of them made each of the other eight positive comments. Furthermore, and most importantly, these comments did not occur until the decrease in the conditioned H-reflex was substantial and the decrease in the control reflex had begun (i.e., the 12th conditioning session and later; Fig. 3).

These spontaneous comments are consistent with the quantitative data showing that successful H-reflex downconditioning was associated with faster walking, more symmetrical walking, better locomotor EMG modulation, and decreased locomotor H-reflexes. By showing that these objective effects were apparent to the subjects in their daily lives, the comments indicate that successful H-reflex conditioning had a substantial practical impact.

\section{Discussion}

This study asked two questions. First, can people with chronic SCI and impaired locomotion decrease the soleus H-reflex in response to an operant conditioning protocol? Second, if so, is H-reflex decrease associated with improved locomotion? The results answer these questions clearly. First, people with incomplete SCI and impaired locomotion decreased the $\mathrm{H}$-reflex in response to an operant down-conditioning protocol (DC subjects). Their success rate and magnitude of reflex change were comparable to those of people who were neurologically normal. H-reflex decrease was specific to the down-conditioning protocol; it did not occur in subjects in whom the H-reflex was simply elicited without feedback (UC subjects).

Second, $\mathrm{H}$-reflex decrease was associated with faster and more symmetrical locomotion. The improvement was evident both in quantitative testing and, most important, to the subjects themselves in their daily lives. It did not occur in people in whom the H-reflex did not decrease, whether they were UC subjects or unsuccessful DC subjects. Indeed, locomotion became less symmetrical in every subject in whom the H-reflex did not decrease. (This greater asymmetry, combined with their slight increase in walking speed, suggests that these subjects expected to be walking faster after the 30 sessions, and thus walked slightly faster by taking more asymmetrical steps.)

These results indicate that reflex-conditioning protocols might help to restore motor function after SCI or in other disorders. They also provide insight into the factors shaping the plasticity associated with $\mathrm{H}$-reflex conditioning and into its potential therapeutic applications.

\section{H-reflex conditioning in people with or without spinal cord injury}

The subjects with SCI were not significantly different from neurologically normal subjects (Thompson et al., 2009) in the probability of successful down-conditioning, and they were identical in the average magnitude of their H-reflex decrease (i.e., 31\%). This finding is consistent with results for soleus H-reflex condi- 
tioning in spinal cord-injured rats (Chen et al., 2006b) and biceps spinal stretch reflex conditioning in people with SCI (Segal and Wolf, 1994).

The subjects with SCI did differ significantly from normal subjects in the composition of their final H-reflex change. Conditioned H-reflex change is composed of task-dependent adaptation (i.e., within-session difference between the control H-reflex and the conditioned H-reflex) plus long-term change (i.e., across-session change in the control H-reflex) (Thompson et al., 2009). The former is thought to reflect immediate change in cortical influence (e.g., on presynaptic inhibition), while the latter reflects spinal cord plasticity. Task-dependent adaptation was significantly less in subjects with SCI than in neurologically normal subjects $(-7 \%$ vs $-15 \%)$, while long-term change was significantly more $(-24 \%$ vs $-16 \%)$. The lesser task-dependent adaptation in subjects with SCI may reflect damage to the corticospinal tract (CST), the spinal cord pathway principally responsible for H-reflex conditioning (Chen et al., 1996, 1999, 2002; Chen and Wolpaw, 2002), and may account for the slightly slower course of H-reflex decrease (i.e., over 30 sessions vs 24 in normal subjects) (Thompson et al., 2009).

The greater long-term change in subjects with SCI is more surprising. It may result from the fact that task-dependent adaptation affects the H-reflex pathway only during the conditioning protocol, while long-term change affects it continuously, and thus has much wider effects. Because the spinal cord serves many behaviors, spinal cord plasticity affects many behaviors. In neurologically normal subjects, the spinal cord plasticity responsible for the long-term change in the H-reflex is likely to disturb behaviors such as locomotion, which are already satisfactory; and it may thereby lead to additional plasticity that compensates for the change in the H-reflex pathway. Animal data support this inference. In normal rats, up- or down-conditioning of the soleus $\mathrm{H}$-reflex increases or decreases, respectively, the soleus locomotor burst, but does not disturb the symmetry of the step cycle, suggesting that plasticity elsewhere preserves this symmetry (Chen et al., 2005). Indeed, in normal rats a conditioned change in the soleus $\mathrm{H}$-reflex is usually accompanied by an opposite change in the quadriceps H-reflex, and also by changes in ankle and hip joint angles during locomotion (Chen et al., 2011). The angle changes are reciprocal and help to ensure that hip height during stance does not change. It appears that in normal rats, and presumably in normal humans as well, compensatory plasticity prevents the plasticity responsible for the modified H-reflex from disrupting normal locomotion.

Furthermore, in normal subjects, the processes that preserve other behaviors may reduce the long-term plasticity that decreases the H-reflex. Wolpaw (2010) hypothesizes that spinal neurons and pathways are maintained in a state of "negotiated equilibrium," a balance that ensures the satisfactory performance of all the behaviors in the individual's current repertoire (Nielsen et al., 1993; Ozmerdivenli et al., 2002; Zehr, 2006). In normal subjects, the spinal cord plasticity underlying

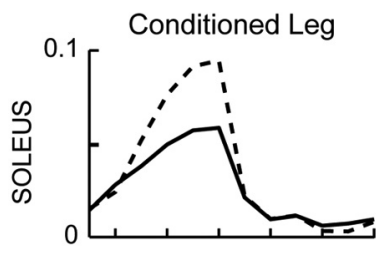

Contralateral Leg
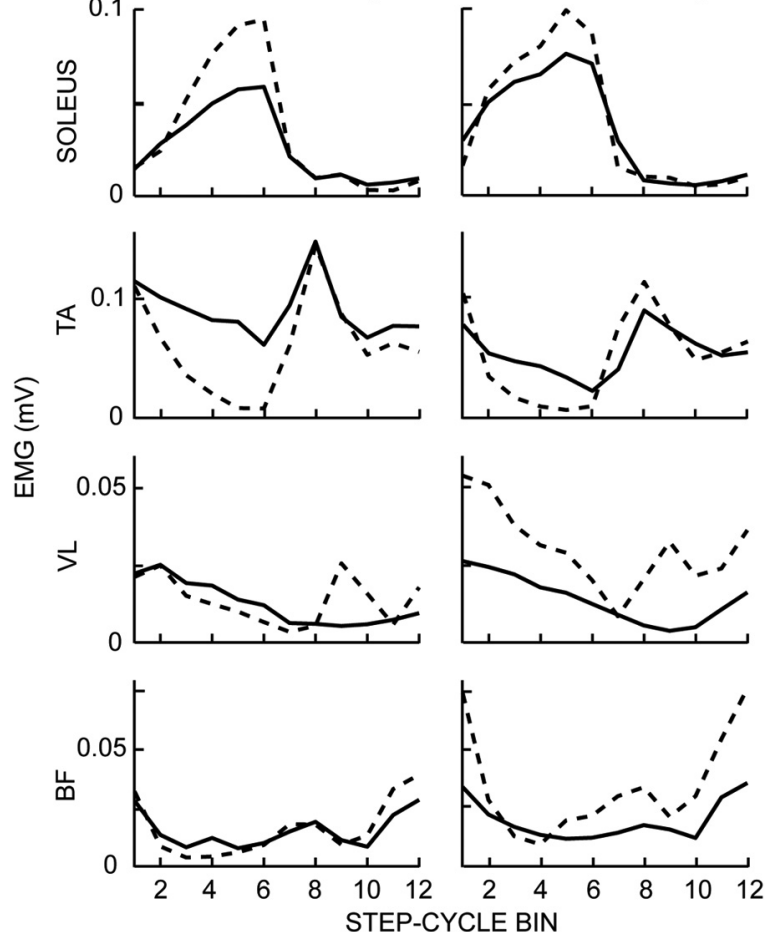

Figure 5. Rectified locomotor EMG activity in soleus, TA, VL, and BF muscles of both legs before (solid line) and after (dashed line) conditioning in a DC subject with $\mathrm{SCl}$ in whom the soleus H-reflex decreased. The step cycle is divided into 12 equal bins, starting from foot contact. Thus, bins 1-7 are for the stance phase and bins 8-12 are for the swing phase. After successful down-conditioning, EMG modulation over the step cycle is greater in both legs.
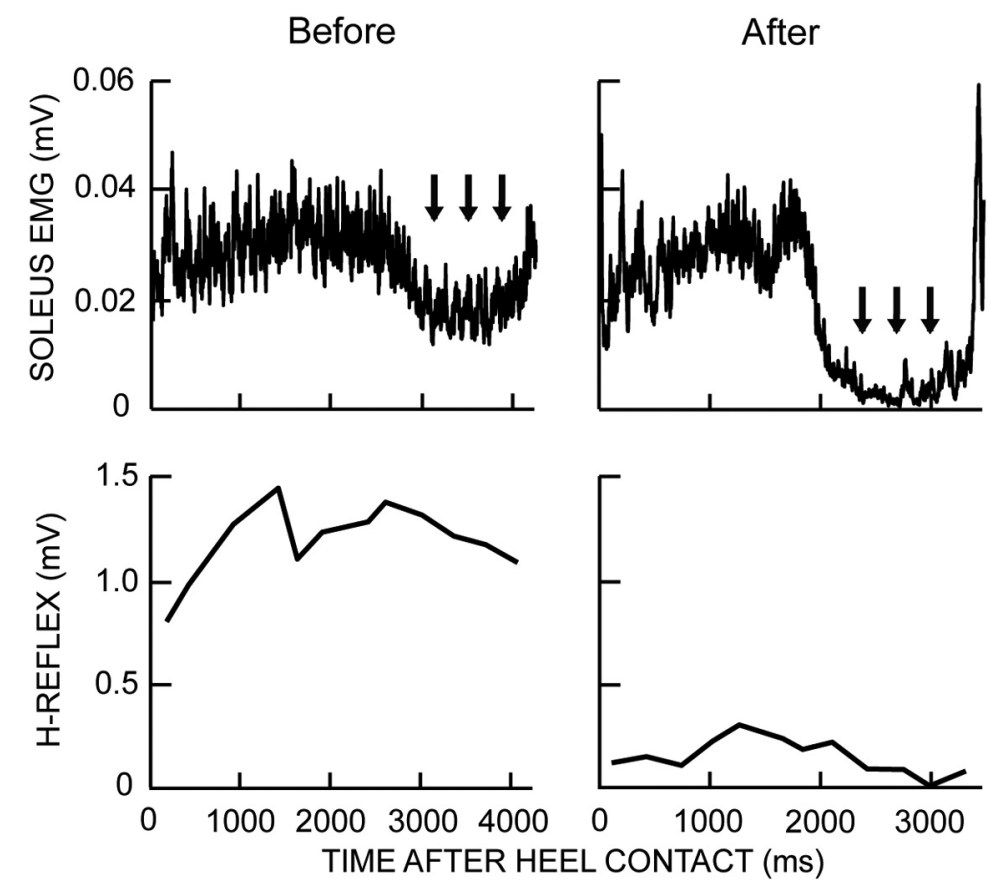

Figure 6. Rectified soleus EMG and locomotor H-reflex size over the step cycle before and after H-reflex down-conditioning in a DC subject with $\mathrm{SCl}$ in whom the H-reflex decreased. The reduced spasticity after conditioning produces better soleus EMG modulation: the abnormal activity during the swing phase (arrows) is no longer present. In addition, the locomotor H-reflex is greatly decreased and better modulated after conditioning (i.e., it is lowest during the swing phase). 


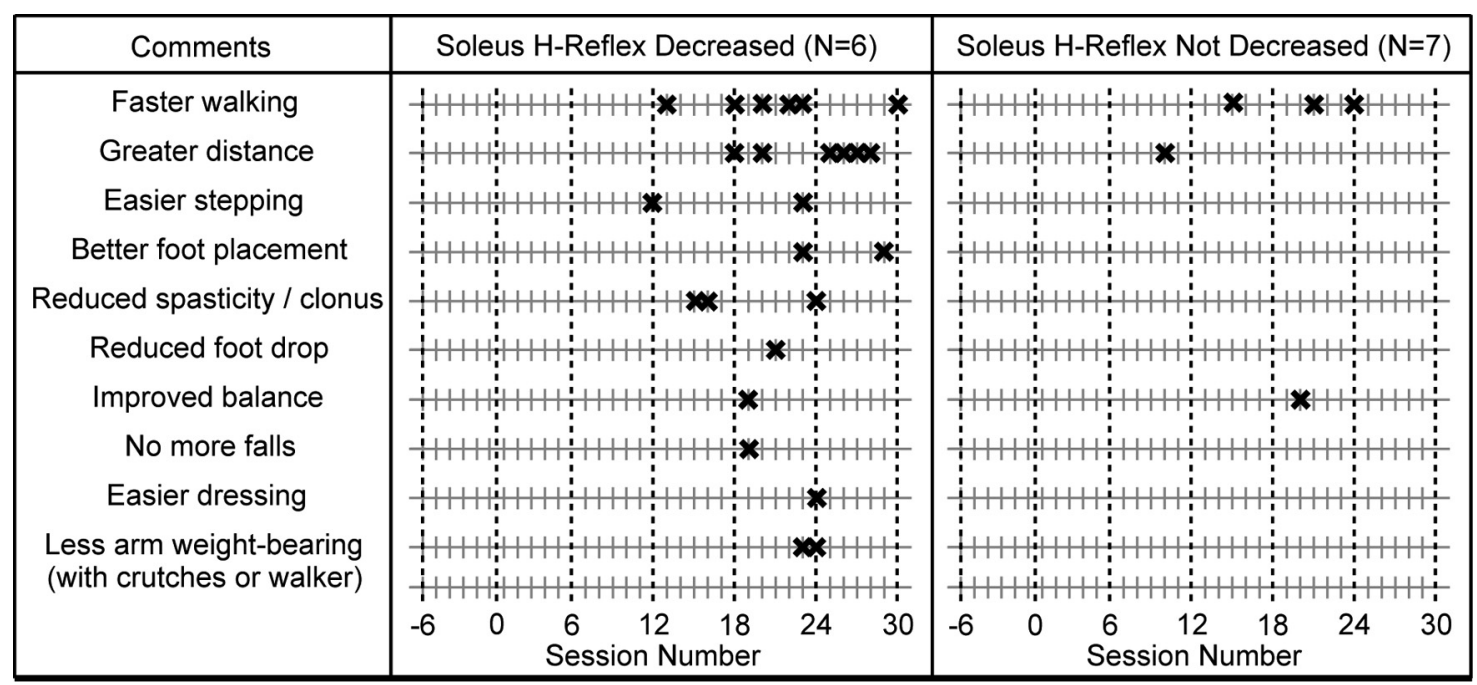

Figure 7. Spontaneous comments made by subjects over the course of data collection. " $X$ " indicates when a subject made the comment for the first time. Note that every subject in whom the H-reflex decreased reported walking faster and farther, and that these reports did not occur until substantial H-reflex decrease had occurred (i.e., Fig. 3).

the new behavior (i.e., a smaller $\mathrm{H}$-reflex) requires the creation of a new equilibrium that produces a smaller H-reflex and still continues to serve other behaviors satisfactorily. This new negotiation produces concurrent adaptive changes in the networks supporting the multiple behaviors that use the pathway. For behaviors such as locomotion, which are already satisfactory, these adaptations are likely to impede the long-term plasticity that decreases the H-reflex. The result is that, in normal subjects, much of the final change in the conditioned H-reflex is due to taskdependent adaptation, which does not disturb other behaviors.

In contrast, for subjects with SCI, the spinal cord plasticity responsible for the long-term H-reflex decrease improves locomotion, an important motor skill. Similarly, in rats in which a spinal cord injury has produced an asymmetrical step cycle, appropriate conditioning of the soleus $\mathrm{H}$-reflex restores step-cycle symmetry (Chen et al., 2006b). In these SCI rats, as in the subjects of this study, the long-term change in the H-reflex is doubly adaptive: it increases reward probability in the conditioning protocol, and it also improves locomotion. It creates a new spinal cord equilibrium superior to the one that prevailed before $\mathrm{H}$-reflex conditioning. In sum, it is probable that long-term $\mathrm{H}$-reflex change is greater in subjects with SCI than in normal subjects because it serves more than the new behavior, it also benefits locomotion.

\section{Potential therapeutic applications of reflex- conditioning protocols}

This study sought to down-condition the soleus H-reflex on the rationale that reducing the activity of this pathway would reduce the hyperreflexia that impaired locomotion in these subjects with incomplete SCI (Dietz and Sinkjaer, 2007; Nielsen et al., 2007). Successful down-conditioning did improve locomotion. Walking became faster and more symmetrical. Furthermore, the locomotor behaviors of knee and ankle extensor and flexor muscles in both legs became more strongly modulated in synchrony with the step cycle, which presumably contributed to the improvement in walking speed and symmetry.

These encouraging results are surprising in their breadth. It is unlikely that the plasticity underlying a smaller soleus $\mathrm{H}$-reflex in one leg could itself have such broad beneficial effects on walking, including increasing the locomotor EMG modulation of con- tralateral muscles. This broad impact, combined with the animal data discussed above, implies that in these subjects with SCI, $\mathrm{H}$-reflex conditioning triggered additional plasticity in other pathways important in locomotion, and thereby changed the entire behavior. These subjects had been injured 0.7-10 years earlier, and their locomotor deficits were stable. In this setting, the acquisition of a new behavior, a down-conditioned soleus $\mathrm{H}$-reflex, disturbed the postinjury equilibrium that the injured spinal cord had reached. It apparently triggered widespread adaptive plasticity that produced a new equilibrium that both decreased the H-reflex and improved locomotion.

Because they can target particular spinal pathways and can either weaken or strengthen the activity of these pathways, reflexconditioning protocols can be designed to focus on each individual's particular deficits. The present study down-conditioned the soleus H-reflex because locomotion was impaired by hyperreflexia. In contrast, the study by Chen et al. (2006b) in spinal cord-injured rats up-conditioned the soleus $\mathrm{H}$-reflex because locomotion was impaired by weak right stance. In both cases, the intervention was effective. This flexibility and specificity are distinctive and desirable features of this new therapeutic approach; and they distinguish it from less focused interventions such as botulinum toxin or baclofen, which simply weaken muscles or reflexes and may have undesirable side effects (Dario and Tomei, 2004; Dario et al., 2004; Sheean, 2006; Ward, 2008; Thomas and Simpson, 2012).

Reflex-conditioning protocols might supplement therapies that involve repetition of complex behaviors [e.g., body-weight supported treadmill training (Edgerton et al., 2008) and constraint-induced movement therapy (Taub and Uswatte, 2003; Wolf et al., 2006)]. Indeed, H-reflex conditioning might be combined with treadmill locomotion so that subjects are rewarded for changing the reflex in a specific phase of locomotion. This combination might help restore normal reflex modulation across the step cycle (Stein and Capaday, 1988). The results of the present study also encourage therapeutic exploration of other reflex-conditioning protocols. For example, in rats, reciprocal inhibition of soleus by common peroneal nerve stimulation can be increased or decreased by operant conditioning (Chen et al., 2006a).

Certainly, the dependence of reflex conditioning on the CST (Chen et al., 1996, 1999, 2002; Chen and Wolpaw, 2002) will 
affect its efficacy in people with SCI. On the American Spinal Injury Association impairment scale (AIS), the disabilities of the present subjects were rated $\mathrm{C}$ or D. Although their success rate was not significantly different from that of normal subjects (Thompson et al., 2009), it was lower; and the extent to which reflex conditioning is possible in people with more severe impairments is unclear. While future improvements in the conditioning protocol may increase success, the need for supraspinal input will remain. On the other hand, reflex conditioning may also prove useful for disorders in which the CST is not affected. For example, in rats in which peripheral nerve transection and reinnervation have produced disordered afferent connections, H-reflex upconditioning can help to restore more normal reflex function (Chen et al., 2010).

\section{Conclusions}

In people with impaired locomotion due to chronic spinal cord injury, down-conditioning of the soleus H-reflex in the more impaired leg was associated with faster and more symmetrical locomotion. This improvement was apparent to the subjects in their daily lives. Similar improvement did not occur in subjects in whom the H-reflex did not decrease. Spinal reflex-conditioning protocols that target each individual's specific deficits might supplement conventional rehabilitation methods and increase functional recovery.

\section{References}

Capaday C, Stein RB (1986) Amplitude modulation of the soleus H-reflex in the human during walking and standing. J Neurosci 6:1308-1313. Medline

Carp JS, Tennissen AM, Chen XY, Wolpaw JR (2006a) Diurnal H-reflex variation in mice. Exp Brain Res 168:517-528. CrossRef Medline

Carp JS, Tennissen AM, Chen XY, Wolpaw JR (2006b) H-reflex operant conditioning in mice. J Neurophysiol 96:1718-1727. CrossRef Medline

Chen XY, Wolpaw JR (1994) Circadian rhythm in rat H-reflex. Brain Res 648:167-170. CrossRef Medline

Chen XY, Wolpaw JR (1995) Operant conditioning of H-reflex in freely moving rats. J Neurophysiol 73:411-415. Medline

Chen XY, Wolpaw JR (2002) Probable corticospinal tract control of spinal cord plasticity in the rat. J Neurophysiol 87:645-652. Medline

Chen XY, Wolpaw JR, Jakeman LB, Stokes BT (1996) Operant conditioning of H-reflex in spinal cord-injured rats. J Neurotrauma 13:755-766. CrossRef Medline

Chen XY, Wolpaw JR, Jakeman LB, Stokes BT (1999) Operant conditioning of H-reflex increase in spinal cord-injured rats. J Neurotrauma 16:175186. CrossRef Medline

Chen XY, Carp JS, Chen L, Wolpaw JR (2002) Corticospinal tract transection prevents operantly conditioned $\mathrm{H}$-reflex increase in rats. Exp Brain Res 144:88-94. CrossRef Medline

Chen XY, Chen L, Chen Y, Wolpaw JR (2006a) Operant conditioning of reciprocal inhibition in rat soleus muscle. J Neurophysiol 96:21442150. CrossRef Medline

Chen Y, Chen XY, Jakeman LB, Schalk G, Stokes BT, Wolpaw JR (2005) The interaction of a new motor skill and an old one: $\mathrm{H}$-reflex conditioning and locomotion in rats. J Neurosci 25:6898-6906. CrossRef Medline

Chen Y, Chen XY, Jakeman LB, Chen L, Stokes BT, Wolpaw JR (2006b) Operant conditioning of $\mathrm{H}$-reflex can correct a locomotor abnormality after spinal cord injury in rats. J Neurosci 26:12537-12543. CrossRef Medline

Chen Y, Wang Y, Chen L, Sun C, English AW, Wolpaw JR, Chen XY (2010) H-reflex up-conditioning encourages recovery of EMG activity and H-reflexes after sciatic nerve transection and repair in rats. J Neurosci 30:16128-16136. CrossRef Medline

Chen Y, Chen L, Wang Y, Wolpaw JR, Chen XY (2011) Operant conditioning of rat soleus $\mathrm{H}$-reflex oppositely affects another $\mathrm{H}$-reflex and changes locomotor kinematics. J Neurosci 31:11370-11375. CrossRef Medline

Dario A, Tomei G (2004) A benefit-risk assessment of baclofen in severe spinal spasticity. Drug Saf 27:799-818. CrossRef Medline

Dario A, Scamoni C, Picano M, Casagrande F, Tomei G (2004) Pharmaco- logical complications of the chronic baclofen infusion in the severe spinal spasticity. Personal experience and review of the literature. J Neurosurg Sci 48:177-181. Medline

Dietz V, Sinkjaer T (2007) Spastic movement disorder: impaired reflex function and altered muscle mechanics. Lancet Neurol 6:725-733. CrossRef Medline

Drew T, Jiang W, Widajewicz W (2002) Contributions of the motor cortex to the control of the hindlimbs during locomotion in the cat. Brain Res Brain Res Rev 40:178-191. Medline

Edgerton VR, de Leon RD, Tillakaratne N, Recktenwald MR, Hodgson JA, Roy RR (1997) Use-dependent plasticity in spinal stepping and standing. Adv Neurol 72:233-247. Medline

Edgerton VR, Leon RD, Harkema SJ, Hodgson JA, London N, Reinkensmeyer DJ, Roy RR, Talmadge RJ, Tillakaratne NJ, Timoszyk W, Tobin A (2001) Retraining the injured spinal cord. J Physiol 533:15-22. CrossRef Medline Edgerton VR, Courtine G, Gerasimenko YP, Lavrov I, Ichiyama RM, Fong AJ, Cai LL, Otoshi CK, Tillakaratne NJ, Burdick JW, Roy RR (2008) Training locomotor networks. Brain Res Rev 57:241-254. CrossRef Medline

Ethier C, Imbeault MA, Ung V, Capaday C (2003) On the soleus H-reflex modulation pattern during walking. Exp Brain Res 151:420-425. CrossRef Medline

Harkema SJ, Hurley SL, Patel UK, Requejo PS, Dobkin BH, Edgerton VR (1997) Human lumbosacral spinal cord interprets loading during stepping. J Neurophysiol 77:797-811. Medline

Hultborn H, Nielsen JB (2007) Spinal control of locomotion-from cat to man. Acta Physiol (Oxf) 189:111-121. CrossRef Medline

Kido A, Tanaka N, Stein RB (2004a) Spinal reciprocal inhibition in human locomotion. J Appl Physiol 96:1969-1977. CrossRef Medline

Kido A, Tanaka N, Stein RB (2004b) Spinal excitation and inhibition decrease as humans age. Can J Physiol Pharmacol 82:238-248. CrossRef Medline

Koski L, Mernar TJ, Dobkin BH (2004) Immediate and long-term changes in corticomotor output in response to rehabilitation: correlation with functional improvements in chronic stroke. Neurorehabil Neural Repair 18:230-249. CrossRef Medline

Lagerquist O, Zehr EP, Baldwin ER, Klakowicz PM, Collins DF (2006) Diurnal changes in the amplitude of the Hoffmann reflex in the human soleus but not in the flexor carpi radialis muscle. Exp Brain Res 170:1-6. CrossRef Medline

Maegele M, Müller S, Wernig A, Edgerton VR, Harkema SJ (2002) Recruitment of spinal motor pools during voluntary movements versus stepping after human spinal cord injury. J Neurotrauma 19:1217-1229. CrossRef Medline

Makihara Y, Segal RL, Wolpaw JR, Thompson AK (2012) H-reflex modulation in the human medial and gastrocnemii during standing and walking. Muscle Nerve 45:116-125. CrossRef Medline

Nielsen J (2002) Motoneuronal drive during human walking. Brain Res Rev 40:192-201. CrossRef Medline

Nielsen JB, Crone C, Hultborn H (2007) The spinal pathophysiology of spasticity—from ac basic science point of view. Acta Physiol (Oxf) 189: 171-180. CrossRef Medline

Nielsen J, Crone C, Hultborn H (1993) H-reflexes are smaller in dancers from The Royal Danish Ballet than in well-trained athletes. Eur J Appl Physiol Occup Physiol 66:116-121. CrossRef Medline

Ozmerdivenli R, Bulut S, Urat T, Ayar A (2002) The H- and T-reflex response parameters of long- and short-distance athletes. Physiol Res 51: 395-400. Medline

Rossignol S, Frigon A (2011) Recovery of locomotion after spinal cord injury: some facts and mechanisms. Annu Rev Neurosci 34:413-440. CrossRef Medline

Segal RL, Wolf SL (1994) Operant conditioning of spinal stretch reflexes in patients with spinal cord injuries. Exp Neurol 130:202-213. CrossRef Medline

Sheean G (2006) Botulinum toxin treatment of adult spasticity: a benefitrisk assessment. Drug Saf 29:31-48. CrossRef Medline

Stein RB, Capaday C (1988) The modulation of human reflexes during functional motor tasks. Trends Neurosci 11:328-332. CrossRef Medline

Taub E, Uswatte G (2003) Constraint-induced movement therapy: bridging from the primate laboratory to the stroke rehabilitation laboratory. J Rehabil Med [41 Suppl]:34-40. Medline

Taub E, Uswatte G, Pidikiti R (1999) Constraint-induced movement ther- 
apy: a new family of techniques with broad application to physical rehabilitation-a clinical review. J Rehabil Res Dev 36:237-251. Medline

Thickbroom GW, Byrnes ML, Archer SA, Mastaglia FL (2004) Motor outcome after subcortical stroke correlates with the degree of cortical reorganization. Clin Neurophysiol 115:2144-2150. CrossRef Medline

Thomas AM, Simpson DM (2012) Contralateral weakness following botulinum toxin for poststroke spasticity. Muscle Nerve 46:443-448. CrossRef Medline

Thomas SL, Gorassini MA (2005) Increases in corticospinal tract function by treadmill training after incomplete spinal cord injury. J Neurophysiol 94:2844-2855. CrossRef Medline

Thompson AK, Chen XY, Wolpaw JR (2009) Acquisition of a simple motor skill: task-dependent adaptation plus long-term change in the human soleus H-reflex. J Neurosci 29:5784-5792. CrossRef Medline

Thompson AK, Chen XY, Wolpaw JR (2012) Soleus H-reflex operant conditioning changes the H-reflex recruitment curve. Muscle Nerve. Advance online publication. doi:10.1002/mus.23620. CrossRef Medline

Ward AB (2008) Spasticity treatment with botulinum toxins. J Neural Transm 115:607-616. CrossRef Medline

Wernig A, Müller S (1992) Laufband locomotion with body weight support improved walking in persons with severe spinal cord injuries. Paraplegia 30:229-238. CrossRef Medline

Wernig A, Nanassy A, Müller S (2000) Laufband (LB) therapy in spinal cord lesioned persons. Prog Brain Res 128:89-97. CrossRef Medline

Wolf SL, Segal RL (1996) Reducing human biceps brachii spinal stretch reflex magnitude. J Neurophysiol 75:1637-1646. Medline

Wolf SL, Winstein CJ, Miller JP, Taub E, Uswatte G, Morris D, Giuliani C,
Light KE, Nichols-Larsen D (2006) Effect of constraint-induced movement therapy on upper extremity function 3 to 9 months after stroke: the EXCITE randomized clinical trial. JAMA 296:2095-2104. CrossRef Medline

Wolpaw JR (1987) Operant conditioning of primate spinal reflexes: the H-reflex. J Neurophysiol 57:443-459. Medline

Wolpaw JR (2010) What can the spinal cord teach us about learning and memory? Neuroscientist 16:532-549. CrossRef Medline

Wolpaw JR, O'Keefe JA (1984) Adaptive plasticity in the primate spinal stretch reflex: evidence for a two-phase process. J Neurosci 4:2718-2724. Medline

Wolpaw JR, Seegal RF (1982) Diurnal rhythm in the spinal stretch reflex. Brain Res 244:365-369. CrossRef Medline

Wolpaw JR, Braitman DJ, Seegal RF (1983) Adaptive plasticity in primate spinal stretch reflex: initial development. J Neurophysiol 50: 1296-1311. Medline

Yen CL, Wang RY, Liao KK, Huang CC, Yang YR (2008) Gait training induced change in corticomotor excitability in patients with chronic stroke. Neurorehabil Neural Repair 22:22-30. CrossRef Medline

Zehr EP (2006) Training-induced adaptive plasticity in human somatosensory reflex pathways. J Appl Physiol 101:1783-1794. CrossRef Medline

Zehr EP, Kido A (2001) Neural control of rhythmic, cyclical human arm movement: task dependency, nerve specificity and phase modulation of cutaneous reflexes. J Physiol 537:1033-1045. CrossRef Medline

Zehr EP, Loadman PM (2012) Persistence of locomotor-related interlimb reflex networks during walking after stroke. Clin Neurophysiol 123:796807. CrossRef Medline 